\title{
A Neighbor-Table-Based Multipath Routing in Ad Hoc Networks
}

\author{
Zhongbang Yao*, Junfeng Jiang*, Pingyi Fan", Zhigang Cao*, Victor O. K. $\mathrm{Li}^{\dagger}$ \\ * The State Key Laboratory on Microwave and Digital Communications, Department of Electronic Engineering, Tsinghua \\ University, Beijing, 100084, China, Email: czg-dee@mail.tsinghua.edu.cn
}

${ }^{\dagger}$ Department of Electrical and Electronic Engineering, The University of Hong Kong, Hong Kong, China, Email: vli@eee.hku.hk

\begin{abstract}
Due to station mobility, the network topology is continuously changing in mobile ad hoc networks. In this paper, we propose a neighbor-table-based multipath routing (NTBMR) protocol to track the dynamic topology changes. Distinguished from prior work on multipath protocols employing disjoint paths, NTBMR does not require the routes to be disjoint. In order to verify the different capabilities against dynamic topology changes for disjoint and non-disjoint multipath routings, we make an attempt to analyze their route reliabilities. Theoretical analysis reveals that non-disjoint multipath routing has higher route reliability when the wireless links are unreliable. In NTBMR scheme, we also present a technique to estimate the mean and variance of the lifetime of a wireless link, which can be used to aid route discovery and maintenance. Simulation results show that our multipath routing scheme is relatively robust in an environment with frequent topology changes and can improve the end-to-end delay and packet delivery ratio performance substantially compared to unipath routing.
\end{abstract}

Keywords-ad hoc networks, unipath routing protocol, multipath routing protocol, source routing protocol

\section{INTRODUCTION}

Since the network topology is continuously changing in Mobile Ad Hoc NETworks (MANET) due to the frequent movements of stations, discovering and maintaining an effective route to a destination station are critical tasks. Although the network topology is continuously changing, the local topology information is relatively stable compared to that of the whole network, as described in $[2,3]$. In this paper, we propose neighbor-table-based multipath routing (NTBMR) which tries to utilize the local topology information. For convenience of exposition, we use path and route interchangeably in the following.

Much work has been done in ad hoc routing. The protocols can be classified into different categories based on different criteria. According to the route discovery mechanism, the routing protocols of ad hoc networks can be classified into three kinds: reactive [1, 3], proactive [3] and hybrid [2] protocols. Based on the number of path to the destination, a routing protocol falls into either the unipath or the multipath category. NTBMR is a hybrid, multipath protocol.
Much work has also been done in multipath routing [4-8], and most of them are extensions of unipath routing. Multipath extensions of AODV are presented in [4-5]. Multiple paths is proved to have longer route life than a single path [6] and the proposed routing protocol is a multipath extension of DSR [1]. The split multipath routing (SMR) [7] scheme focuses on discovering and maintaining multiple maximally disjoint paths. Diversity coding is utilized in [8], in which the data packets are fragmented into smaller blocks and distributed to the multiple paths simultaneousiy. Most of the prior multipath routing schemes prefer disjoint routes to the destination. However, there is no analysis to evaluate the route reliability for the two kinds of routings - disjoint and non-disjoint routings. In our paper, we try to introduce the route reliability analysis to evaluate the different performance under unreliable wireless links of both disjoint and non-disjoint multipath routings.

Focusing on combating the frequent topology changes in ad hoc networks, NTBMR does not require the multiple routes to be disjoint. In NTBMR, every station maintains one neighbor table which records its $k$-hop neighbor stations. Although we can get more topology information if we increase $k$, the incurred overhead will increase too. Besides, the reliability of topology information will decrease. Thus, drawing a balance between the correctness and overhead, we limit $k$ to 2 in our scheme.

The rest of this paper is organized as follows. Section II presents the NTBMR scheme. Route reliability analysis is introduced in Section III. Some simulation results are presented in Section IV. We give our conclusions in Section V.

\section{NEIGHBOR TABLE BASED MULTIPATH ROUTING (NTBMR) SCHEME}

NTBMR consists of the following two processes: route discovery and route maintenance. The central mechanism in our scheme is how to maintain a neighbor table and a route cache in every station. The routes in the neighbor table are not only used directly in the establishment of route cache but also 
used to estimate the lifetime of wireless links to aid route discovery.

\section{A. Establishment of Neighbor Table and Route Cache}

In NTBMR, every station in the network periodically transmits beacon packets. Using the time-to-live (TTL) field as a counter, these packets will only be transmitted among its two-hop neighbors. The neighbor table is established based on the route information in the beacon packet. Each beacon packet contains the following fields: packet type, source address, intermediate station address, unreachable station address, TTL, and sequence number. The neighbor table can be established by time-driven and/or data-driven mechanisms, described as follows.

Under the time-driven mechanism if a station receives the beacon packet along one specific route, the station considers the route active and adds all the stations the packet has passed by to the neighbor table. That is, the one-hop neighbor can obtain one-hop route to the source transmitter, and the two-hop neighbor station obtains a two-hop route to the source transmitter besides a one-hop route to the intermediate relay station. But if the station cannot receive the beacon packet along the route within a predefined timeout period, it regards that route inactive and eliminates the corresponding stations along the route from the neighbor table. The main disadvantage of this mechanism is that the station cannot know the actual topology changes during this timeout period, i.e., there is a time lag before the topology changes are sent to the related stations.

This disadvantage of the time-driven mechanism can be alleviated through the "data-driven" mechanism. This is accomplished by utilizing one field of the beacon packet. Once a station detects its one-hop station is unreachable, it will fill the field with the address of the unreachable station to inform its other one-hop neighbor stations to update their neighbor table when transmitting the beacon packet next time. As soon as the other one-hop neighbors receive the beacon packet, it will delete the "unreachable station" contained in the beacon packet from its two-hop neighbors in the neighbor table. The detection of one-hop unreachable stations can be achieved by the link failure detection mechanism of the media access control (MAC) layer or the timeout of the beacon packets transmitted by the unreachable stations.

Route cache is used for route discovery and maintenance. It contains all the routes that the station knows about. Whenever the neighbor table changes, the corresponding items in the route cache will also be updated in order to track the actual network topology changes. In addition, to reduce the number of route discovery processes, the route cache will be updated by monitoring the route information contained in other packets besides the route reply packets, such as route request packets, route error packets and data packets. To differentiate the routes, we set different priorities for routes which are obtained through different methods. We call this route extraction reason. The routes extracted from reply packets have the highest priority, routes from request packets or neighbor table take the second priority and routes from data packets have the lowest priority. These priorities will be used to aid route selection.

\section{B. Lifetime Parameter Estimation of Wireless Links}

The neighbor table is used to modify and refresh the local routes in the route cache described above. In addition, it can be used to estimate some parameters of the network. NTBMR utilizes the neighbor table to estimate the mean and variance of the wireless link lifetime using the following expressions,

$$
\begin{gathered}
M \leftarrow \alpha M+(1-\alpha) M_{c u r} \\
V \leftarrow \beta V+(1-\beta) V_{c u r}
\end{gathered}
$$

where $M, V$ are the mean and variance of the wireless link lifetime, $M_{c u r}, V_{c u r}$ are the lifetime of the current broken wireless link and its corresponding variance, and $\alpha, \beta$ are two parameters with $0<\alpha, \beta<1$. Every station computes $M$ and $V$ based on the one-hop topology changes in the neighbor table. During route discovery, the source station will determine if one route is usable or not based on $M$ and $V$.

\section{Route Discovery}

When the data packet from an upper layer arrives, the station firstly tries to find one effective route from its route cache. Since there may exist multiple routes to the same destination, we should carefully design the criterion for route selection.

Most of the prior research prefers the route over which the destination receives the route request packets first [4-7], i.e., the primary route. In our scheme, we select the route based on multiple attributes, including route setting up time, route distance, route extraction reason, etc. We denote the attributes of route $i$ as the following expression,

$$
A_{i}=\{[t], d, r\}
$$

where $t$ represents the route setting up time, $d$ represents hop count from source to destination, $r$ represents the route extraction reason and $[t]$ denotes the maximal value in grids of 10 millisecond which is not larger than $t$. NTBMR uses a joint route selection criterion utilizing $\left\{A_{i}, i \in B\right\}$, where $B$ denotes the set of routes having the same destination in the route cache. The priority of $\{[t], d, r\}$ is assigned in decreasing order when selecting routes. That is, the station firstly selects the route with the greatest $[t]$ value under the condition that the elapsed time since $t$ does not exceed $M+V$, then the one with the shortest distance to the destination is selected. If more than one route have the same [ $t]$ and $d$ values, the station will select based on the route extraction reason.

If the station cannot find an eligible route to the destination, the station will initiate the route discovery process which is similar to DSR [1]. After the station selects one route 
to the destination, it will fill the station addresses of the route to the corresponding fields of the data packet. Then the intermediate stations can relay the packet based on these fields.

\section{Route Maintenance}

If a route fails during transmission, the eligible altemate routes will be used. An intermediate station encountering link failure will take different actions according to two predefined transmission time threshold values $T_{1}$ and $T_{2}$ with $T_{1}<T_{2}$, which is similar to the DSR simulation model in [9]. This process is shown in Fig. 1.

If the intermediate station receives a route error packet, it will update its neighbor table and route cache based on the route error information. Then it will transmit the route error packet to its upstream station. As soon as the source station receives the route error packet, it firstly modifies the neighbor table and route cache. Then it will initiate a new route discovery process described above if it still has data packets to transmit.

$$
\begin{aligned}
& \text { If (re-transmission times } \left.<=T_{1}\right) \text { then transmits again; } \\
& \text { Elseif (re-transmission times } \left.<=T_{2}\right) \text { then } \\
& \text { 1) Finds another eligible route to the same destination; } \\
& \text { 2)Transmits route error packet to the upstream station. } \\
& \text { Else } \\
& \text { 1) Discards the packet; } \\
& \text { 2) Transmits route error packet to the upstream station. } \\
& \text { End If }
\end{aligned}
$$

Fig. 1. Actions Taken by the Intermediate Stations when Enoountering Route Failure

\section{ROUTE RELIABILITY ANALYSIS}

In [6], A. Nasipuri and S. R. Das proposed an analytical modeling framework to determine the time interval between successive route discoveries. We use a similar analysis technique to analyze the route reliability of disjoint and nondisjoint multipath routings under unreliable wireless links.

A route from $S$ to $D$ consisting of a sequence of $k$ wireless links is expressed as a random variable $R$ and the associated wireless links are $\left\{L_{i},(i=1,2, \ldots, k)\right\}$. The lifetime of $L_{i}$ and $R$ are denoted by $T_{L}$ and $T_{R}$, respectively. Assuming that $\left\{T_{L_{i}},(i=1,2, \ldots, k)\right\}$ are independent and identically distributed ( i.i.d) exponentially distributed random variables with the same parameter $T$, the probability density function of the lifetime of a wireless link $L_{i}$ can be expresses as follows,

$$
f(t)_{4}=T e^{-r t} \quad(i=1,2, \ldots, k) .
$$

As we know, if any wireless link of a route is broken, the route will fail. Thus we get

$$
T_{R}=\min \left(T_{h}, T_{L_{2}}, \ldots, T_{L_{2}}\right) \text {. }
$$

We define the reliability of a route as follows,

$$
R=P\{X>t\}(t>0),
$$

where $X$ is the lifetime of the route.

Let $F(X)$ denote the cumulative distribution function of $X$, we have

$$
R(X)+F(X)=1 .
$$

In fact, the reliability of a route refers to the probability that stations can utilize this route to transmit data successfully. When we regard the routes having the same hop number as "Virtual Links" (VL), the above analysis method is still valid. In the following, we shall consider two situations when transmitting data packets. One uses the disjoint multipath routing protocol and the other uses the non-disjoint multipath routing protocol. (shown in Fig. 2.) In Fig. $2 V L_{i j}$ is a virtual wireless link connecting stations $i$ and $j$. Suppose that the lifetime of every link can be represented by a random variable $T_{V_{L_{i j}}},(i=1,2, \ldots, m, j=1,2, \ldots, n)$.

The lifetime of the route from $S$ to $D$ is

$$
\begin{aligned}
& T_{\text {dqjir }}(S \rightarrow D)=\max \left\{\min \left\{T_{Y_{41}}, \ldots, T_{V_{H_{n}}}\right\}, \ldots, \min \left\{T_{V_{n_{m 1}}}, \ldots, T_{V_{m n}}\right\}\right\}
\end{aligned}
$$

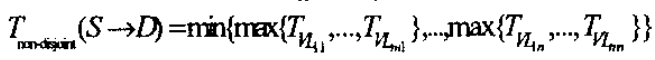

Since we assume all the links follow the same cumulative distribution function $F_{T_{V L}}(t)$, the cumulative distribution functions of the two different multipath protocols are expressed as

$$
\begin{gathered}
F_{\text {disjoint }}(t)=\left[1-\left(1-F_{T_{I 2}}(t)\right)^{n}\right]^{m}, \\
F_{\text {non-disjoint }}(t)=1-\left[1-\left(1-F_{T_{12}}(t)\right)^{m}\right]^{n} .
\end{gathered}
$$

Thus we get the reliability as follows,

$$
\begin{aligned}
& R_{\text {disjoint }}(t)=1-\left[1-\left(1-F_{T_{V^{\prime}}}(t)\right)^{n}\right]^{\mathrm{n}} \\
& R_{\text {non-disjoint }}(t)=\left[1-\left(1-F_{T_{V_{L}}}(t)\right)^{m}\right]^{\mathrm{n}} .
\end{aligned}
$$

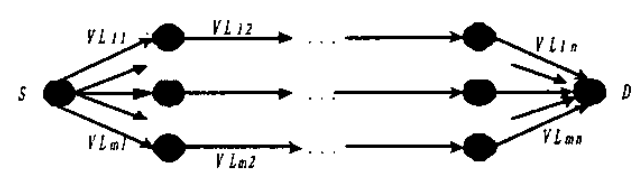

(a) Disjoint loutes

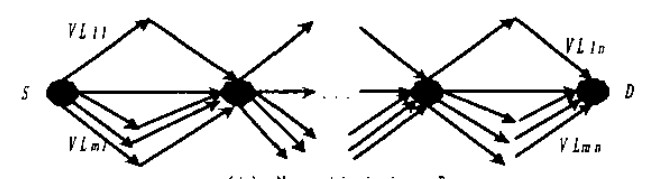

(b) Non-disjoint Routes

Fig. 2. Demonstration of Disjoint and Non-disjoint Routes between Source and Destination Stations

Fig. 3 illustrates the theoretical route reliabilities of the two different multipath routing protocols, where the same $F_{T_{n}}(t)$ value is employed. It can be seen that the non-disjoint 
multipath routing protocol outperforms the disjoint multipath routing protocol. The main reason is that non-disjoint multipath routing can provide more redundancy in the unreliable wireless links environment.



Fig. 3. Performance Comparison of Disjoint and Non-disjoint Multipath Routing Protocols ( $m=3$ )

\section{Performance EVAluation}

We evaluate NTBMR performance through simulations. In the simulations, 20 sources generate CBR traffic $(512$ bits/packet and 4 packets/second) of 50 stations moving in a $600 \mathrm{~m} \times 600 \mathrm{~m}$ area according to the "random way-point" model. We assume that every station has the capability to transmit and receive radio signals in a radius of $150 \mathrm{~m}$. The source station randomly selects one of the 50 stations as its destination. The simulation period is 600 seconds and the results are the averages of five simulations with different random seeds. IEEE 802.11 MAC and physical protocols are adopted and the channel bit rate is $2 \mathrm{Mbps}$. The transmission period of the beacon packet is set to be one second. The predefined retransmission times are set to be $T_{4}=2$ and $T_{2}=5$, respectively. $\alpha$ and $\beta$ are both selected as 0.8 .

For comparison between NTBMR and other routing protocols, we use DSR [1] and SMR [7] as the reference routing protocols. In DSR, the source transmits RREQ packet and the intermediate stations, when receiving the RREP packet, will save the routes contained in the RREP packet. Furthermore, the intermediate stations do not relay the received duplicate RREQ packets, but they can transmit the RREP packet instead of the destination if they can find one route to the destination in their route table. But in SMR, the source floods RREQ packet and the intermediate stations repeatedly relay RREQ if the duplicate packets traverse through a different incoming link than the link from which the first RREQ is received, and whose hop count is not larger than that of the first received RREQ [7]. Only the source saves the routes in the RREP. Besides, in order to obtain maximally disjoint paths to the destination, the intermediate stations are not permitted to transmit RREP packets and the destination can reply the RREQ twice. So the source can get two routes to the destination - one is the primary route and the other is the backup route. We make some minor modifications to SMR-1 [7] and come up with V-SMR (Variant SMR), which will be used in the simulations. These modifications include: the two routes saved by the source must be disjoint routes, and the data packets which have not been transmitted before can be sent using either of the two routes and they can be transmitted simultaneously, but the re-transmissions of packets will just use the primary route. We simulate NTBMR with different beacon timeout factors. In NTBMR, the beacon timeout period is $n \times T_{b}$ in which $n$ is called the beacon timeout factor and $T_{b}$ is the beacon transmission period.

Fig. 4 shows the packet delivery ratio of NTBMR, DSR and VSMR. In the simulations, the packet delivery ratio is defined as follows,

$$
P_{d}=\frac{\text { total number of packets the destinations successfully received }}{\text { total number of packets the sources transmitted }} .
$$

Clearly, the packet delivery ratio decreases dramatically as the station traveling speed increases for DSR. However, the influence of station traveling speed is not significant and the impact of different beacon timeout factors on the packet delivery ratio is minimal for NTBMR. The packet delivery ratio of V-SMR is between DSR and NTBMR.

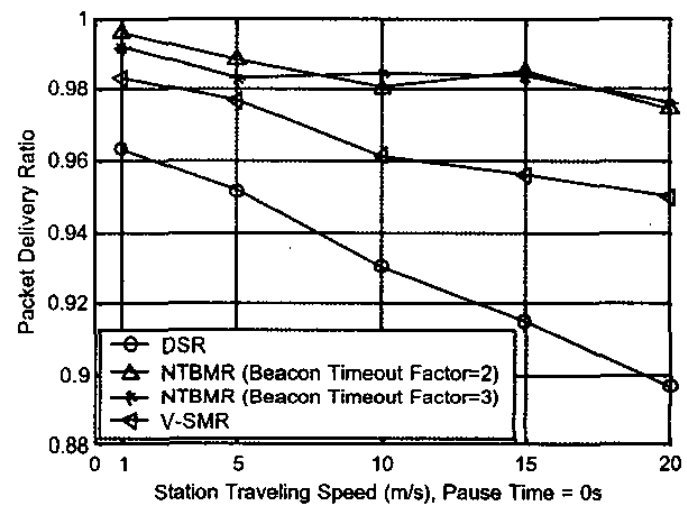

Fig. 4. Packet Delivery Ratio vs. Station Mobility

The average end-to-end transmission delay of data packets is shown in Fig. 5. Obviously, when the station traveling speed increases, the end-to-end delay will increase also for NTBMR, DSR or V-SMR routing. But the increasing rates of DSR and V-SMR routing are higher than that of NTBMR as the station traveling speed increases.

Fig. 6 depicts the transmitted request numbers of NTBMR, DSR and V-SMR. The transmitted request numbers of DSR and V-SMR are significantly higher than that of NTBMR, especially when the station traveling speed is high. This is due to the more powerful capability of NTBMR to combat dynamic topology changes. Since a failure in any of the two disjoint routes causes the source to transmit RREQ packet, the transmitted requests of V-SMR is more than that of DSR. 
Fig. 7 shows the average overhead comparison of NTBMR, DSR and V-SMR. The overhead is computed as the ratio of the total bits contained in the control packets and data packet headers to the data packet bits which arrive from the application layer. The control packets include: beacon packets, route request packets, route reply packets, and route error packets. Fig. 7 indicates that the overhead difference of NTBMR, DSR and VSMR becomes smaller as the station traveling speed increases.

\section{CONCEUSIONS}

In this paper, we propose NTBMR which aims at combating the frequent topology changes in ad hoc networks. Distinguished from most prior research, NTBMR does not require the multiple routes to be disjoint. To compare the route reliability of disjoint and non-disjoint multipath routing, we introduce an analysis model. The theoretical results have validated the effectiveness of non-disjoint multipath routings. In NTBMR, we not only use the neighbor table to update the routes in the route cache but also use it to estimate the mean and variance of the lifetime of the wireless links, which can aid in route discovery. When selecting one effective route from the multiple routes in the route cache, we propose a joint route selection criterion which utilizes multiple attributes of the routes - setting up time, distance to the destination, and route extraction reason.

Simulation results have shown that the NTBMR protocol are superior in terms of packet delivery ratio, end-to-end delay and transmitted request number compared with DSR and $V$ SMR routings. But they are achieved at the expense of additional overhead which mainly results from the periodic transmission of beacon packets.

\section{ACKNOWLEDGMENT}

This research is supported in part by the National Natural Science Foundation of China/Research Grants Council of Hong Kong Joint Research Scheme under Grant No. N_HKU $009 / 00$ and No. 6001161943.

\section{REFERENCES}

[1] J. Broch, D B. Johnson, D A. Maltz. "The Dynamic Source Routing Protocol for Mobile Ad Hoc Networks, "Internet Draft, draft -ietfmanet dsr-*.txt.

[2] Z. J. Haas, M. R. Pearlman. "The Zone Routing Protocol for Ad Hoc Networks," Internet Draft, draft-ietf-manet-zone-zrp-*.txt.

[3] A. Iwata, C C. Chiang, G Y. Pei, M. Gerla, T. W. Chen, "Scalable Routing Strategies for Ad Hoc Wireless Networks" IEEE JSAC, Vol. 17, No. 8, Aug. 1999

[4] S. J. Lee, M. Gerla, "AODV-BR: Backup Routing in Ad Hoc Networks," IEEE WCNC 2000, Page 1311-1316.

[5] M. K. Marina S. R. Das, "On-demand Multipath Distance Vector Routing for Ad Hoc Networks" Proceedings of IEEE ICNP, Riverside, CA, Nov. 2001.

[6] A. Nasipuri, S. R Das " On-Demand Multipath Routing for Mobile Ad Hoc Networks" Proceedings of IEEE ICCCN'99, Boston, MA, Oct. 1999, pp. 64-70.

[7] S. J. Lee, M. Gerla, "Split Multipath Routing with Maximally Disjoint Paths in Ad Hoc Networks," ICC 2001.
[8] A. Tsirigos, Z. J. Haas, "Multipath roting in the presence of frequent topological changes," IEEE Communications Magazine, Vol. 39,Nov. 2001

[9] J. T. Ballah, "Integrated MANET Mutual Authentication System (IMMAS)," Master's thesis, Air Force Institute of Technology, March 2002

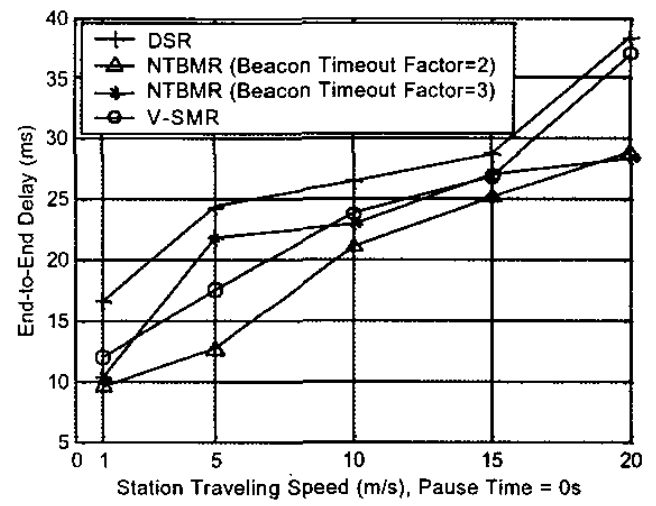

Fig.5. End-to-End Delay vs. Station Mobility

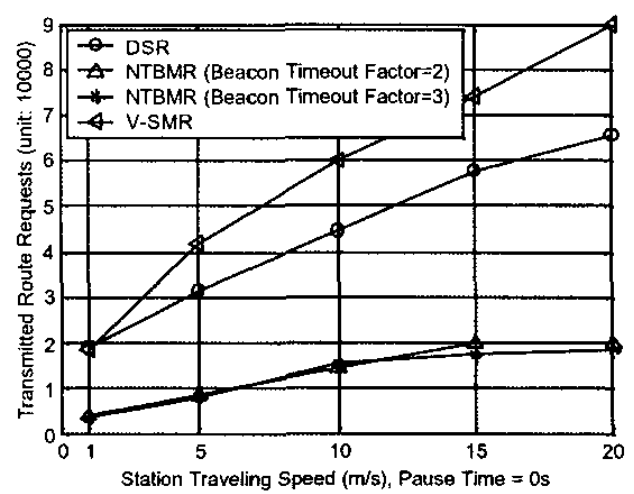

Fig. 6. Transmitted Route Requests vs. Station Mobility

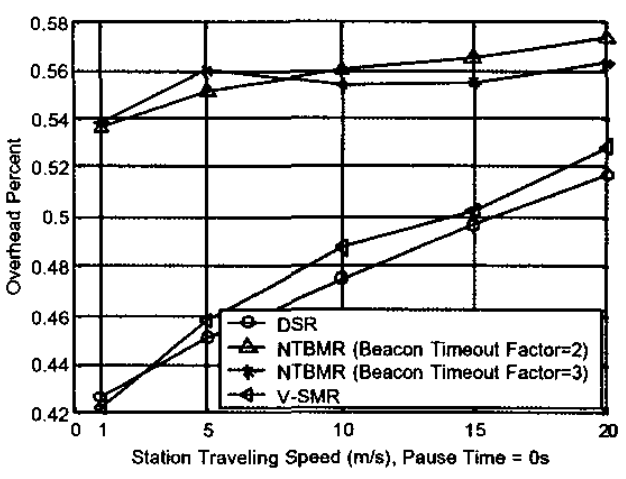

Fig. 7. Overhead vs. Station Mobility 